\title{
The Physical Society Annual Exhibition
}

$\mathrm{Y}$ EAR by year the Physical Society holds an exhibition of scientific instruments and apparatus, which has now grown so that this year-the twenty-ninth-111 individuals and firms describe their exhibits in a catalogue of more than two hundred pages. The exhibition is divided into two sections, one a Trade Section where various physical instruments may be inspected, and the other a Research and Experimental Section.

The Trade Section is of great value to the prospective purchaser of physical apparatus, since it gives him the opportunity of comparing alternative makes of instrument at one time. This aim would be facilitated by a better index to the catalogue so that, for example, gas-analysis apparatus and carbon dioxide and oxygen meters came under a common heading. One reflection that must strike the visitor to this assemblage of instruments is the immense variety of uses to which electronic devices are now put in commercial and industrial instruments. British industry also responds quite rapidly to new needs imposed by legislation or by the political situation, and A.R.P. is mentioned in several of the catalogue descriptions. The Factories Act of 1937 has led to a spurt in the output of photometric equipment, much of which utilizes photo-electric cells, thereby obtaining rapid response and the advantage of a wide range; one instrument can read from extremely low values, of the order of $0 \cdot 001$, up to $100 \mathrm{ft}$.-candles. The photo-electric cell finds application also in temperature control. For this purpose, the pointer of the instrument which indicates the temperature to be controlled casts a shadow on a selenium cell, and any deviation from its pre-selected position operates the cell and hence a control to restore the temperature to its original value.

The report of the Ministry of Transport Committee on traffic noise is doubtless a contributory factor to the activity shown in the production of noise-meters and other acoustic instruments, of which many were on view. The high-vacuum pump has long been an important tool both in the physical laboratory and in technical processes, and it was interesting to see a new diffusion pump in which the oil or mercury had been replaced by an organic fluid of very low vapour pressure, and means had been provided for continuous self-purification of this fluid, thus avoiding the accumulation of condensable vapours in the pump system, and permitting the attainment of very high vacua, whatever the composition of the gases being removed.

Turning attention now to the Research Section, it is impossible to deal in detail with all the exhibits, which range over almost the whole field of pure and applied physics. Some of the best, such as the colorimetric equipment of Dr. W. D. Wright and Mr. H. V. Walters, and the investigations on dielectrics by the staff of the British Electrical and Allied Industries Research Association, were of rather specialist character. An exhibit of more general interest was provided by the Physics Department of the National Physical Laboratory, and illustrated forcibly the great variety of problems dealt with at the Laboratory. The acoustics exhibit in- cluded the analysis of noise, where visitors could see the intensities of the various frequency components in a complex sound as a linear spectrum in a cathode ray oscillograph, as well as the wave-form of the same sound in a neighbouring cathode ray tube; models also demonstrated how the transmis sion of noises in a building can be diminished. Other exhibits showed the work on radium and $\mathrm{X}$-rays, whilst a set of illuminated models of crystal lattices was attractive, even if it conveyed little new information.

Many visitors were attracted by the exhibit organized by the Scientific Computing Service, Ltd., where in addition to a display of ealculating machines, there was a new 135-character typewriter, and an interesting survey of the whole history of the production of a mathematical table, from the first calculations to the final printing. Pure mechanics is somewhat out of fashion, but Messrs. W. and T. Avery, Ltd., showed that it still has points of interest, in an exhibit which showed various little-known statical principles which find application in weighing mechanisms. Messrs. H. A. Boot and A. H. Knight showed a small model of the electrostatic generator used in nuclear research by Prof. van de Graaff, which operates by collecting charges from a moving insulated belt and allowing these to accumulate within a metal sphere. The generator shown could furnish a current of $30 \mu a .$, the maximum voltage being 250,000 .

Two ingenious experiments for the school laboratory were shown by Mr. W. O. Clarke, in one of which Planck's constant is determined with apparatus no more complicated than a commercial photometer and an incandescent lamp with electrical measuring instruments, and the other enables the specific heat of air to be measured by using a hot-air blower as the basis of a continuous-flow calorimeter.

Among the many novelties shown by the Research Laboratories of the General Electric Co., Ltd., was an apparatus for measuring the mean strength of a magnetic field by amplifying the alternating E.M.F. obtained when a small coil is rotated in the field. They also demonstrated a method by which the intensity of the light from a photo-flash lamp (which reaches its maximum in only 0.05 sec. from ignition) can be studied in detail as a function of time.

In a demonstration by Drs. E. E. Widdowson and S. J. Gregg, multi-molecular layers of barium stearate were being built up by the successive deposition of mono-molecular layers. A striking feature is the vivid colours, changing rapidly with the number of layers, which are seen when the multi-molecular film is viewed by polarized light. The behaviour of lamp filaments and their mountings under vibration must be important to the designer and manufacturer, and many visitors found a stroboscopic picture of this behaviour to be both attractive and informative. This was one of several items included in the exhibit of the Research Laboratories of the British ThomsonHouston Co., Ltd. Finally, many lecturers will be grateful for the simple method demonstrated by Prof. J. D. Bernal, whereby the relation between the Laue diagram and the Bragg reflection patterns can be shown visually. 